\title{
Nuclear Shell Model Calculations with Non-Loca! Interactions
}

\author{
S. A. Moszkowski \\ Department of Physics \\ UCLA, Los Angeles, CA 90024
}

\author{
S. D. Bloom \\ D. A. Resler
}

Lawrence Livermore National Laboratory

Livermore, CA 94550

This paper was prepared for submittal to

Capture Gamma-Ray Spectroscopy 7th Int. Sym.

Asilomar, CA, October 14-16, 1990

October 14,1990

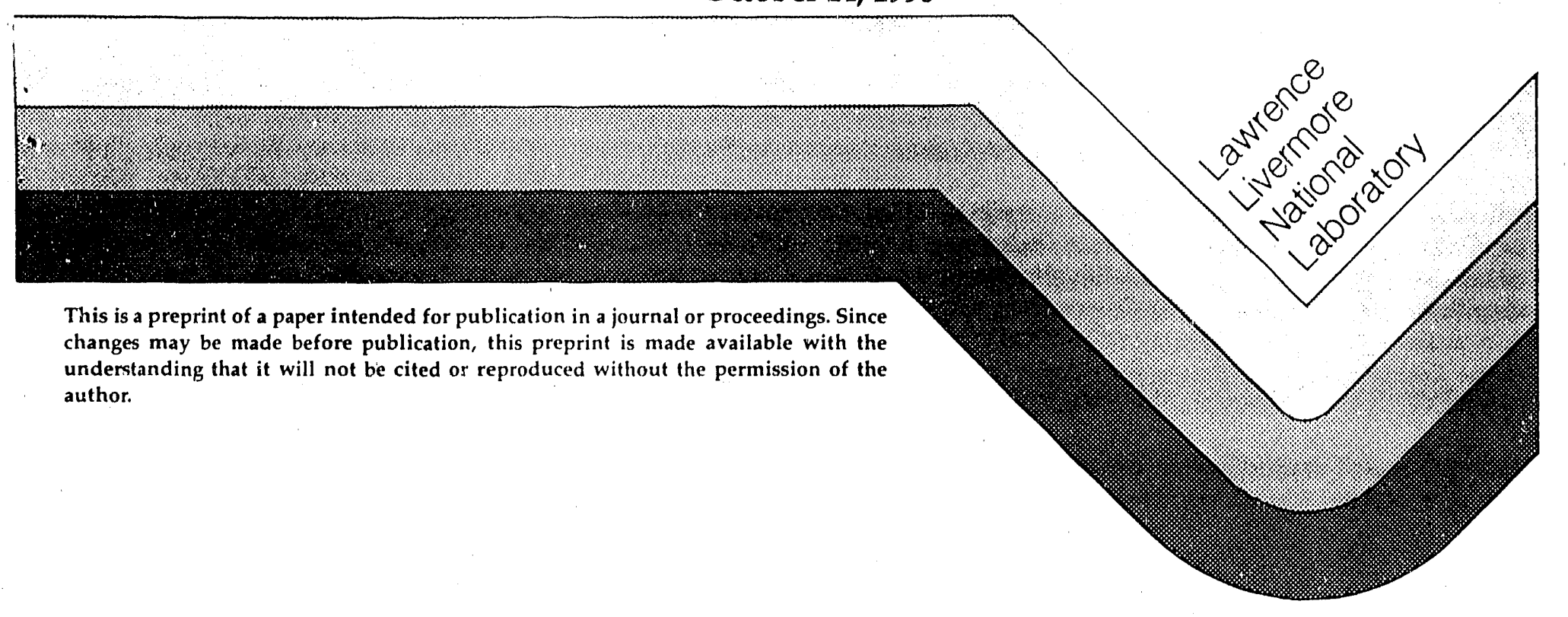

This is a preprint of a paper intended for publication in a journal or proceedings. Since changes may be made before publication, this preprint is made available with the understanding that it will not be cited or reproduced without the permission of the author.

\section{MSTER}




\section{DISCIAIMER}

This document was prepared as an account of work sponsored by an agency of the United States Government. Neither the United States Government nor the University of California nor any of their employees, makes any warranty, express or implied, or assumes any legal liability or responsibility for the accuracy, completeness, or usefulness of any information, apparalus, producl, or process disclosed, or represents that its use would not infringe privately owned rights. Reference herein to any specific commercial products, process, of service by trade name, Irademark, manufacturer, or otherwise, does not necessarily constifute or imply its endorsement, recommendation, or favoring by the Inited States Government or the University of California. The views and opinions of authors expressed herein do not necessarily state or reflect those of the United States Government or the University of Californiz, and shall not be used for advertising or product endorsement purposes. 
INVITED PAPER AT THE "CAPTURE GAMMA-RAY SPECTROSCOPY" 7TH INTERNATTONAL SYMPOSIUM, ASILOMAR, CALIF., 10/14-19/1990

NUCLEAR SHELL MODEL CALGULATIONS WITH NON-LOCAL INTERACTIONS

S.A. Moszkowski

Department of Physics, UCLA, Los Angeles, CA 90024

S.D. Bloom and D.A. Resler

Lawrence Livermore National Laboratory, Livermore, CA 94550

\section{ABSTRACT}

It is becoming clearer with time that non-locality of the nucleon-nucleon interaction can play a significant role in nuclear properties. In this talk we review evidence for such nonlocality. Then, using a Gaussian interaction, we discuss the effect of non-locality on two body matrix elements in the nuclear shell model. Finally, we mention some applications. For example, non-locality leads to faster convergence of off-diagonal matrix elements.

\section{WHAT IS NON-LOCALITY?}

1. Definition

Let us begin with discussing what non-locality is. Basically, the Schroedinger equation for a particle in a conventional.

(local) potential has the well known form:

$-\left(\hbar^{2} / 2 m\right) \psi(\vec{r})+V(\vec{r}) \psi(\vec{r})=E \psi(\vec{r})$.

which can also be written as:

$-\left(h^{2} / 2 m\right) \psi(\vec{r})+\int V(\vec{r}) \delta\left(\vec{r}-\vec{r}^{\prime}\right) \psi\left(\vec{r}^{\prime}\right) d^{3} r^{\prime}=E \psi(\vec{r})$.

For a non-local interaction, we have, instead:

$-\left(h^{2} / 2 m\right) \psi(\vec{r})+\int V\left(\vec{r}, \vec{r}^{\prime}\right) \psi\left(\overrightarrow{r^{\prime}}\right) d^{3} r^{\prime}-E \psi(\vec{r})$.

i.e. $\vec{r}$ and $\vec{r}^{\prime}$ don't have to be at the same point.

2. Relation to momentum dependence

Instead of writing the interaction as a non-local operator, we can express it in the form of a local, but momentum dependent interaction. Thus, for example, a non-local Gaussian interaction of the form:

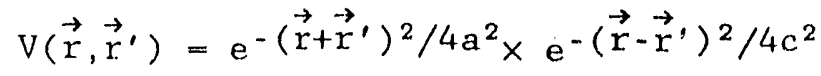

is equivalent (apart from a multiplicative constant) to a momentum dependent Gaussian interaction of the form:

$V(\vec{r}, \vec{p})=e^{-r^{2} / a^{2}} \times e^{-p^{2} c^{2}}$ 
(Actually, this interaction has to be properly symmetrized w.r.t. $r$ and $p$, so that it is Hermitean.) The connection between non-locality and momentum dependence is more complicated for a non-Gaussian interaction.

3. Relation to energy dependence

Instead of writing the interaction as momentum dependent, we can also try to represent it as local, but energy dependent. However, when we do this, we lose the orthogonality property of wavefunctions of different energy.

\section{WHY DO WE NEED NON-LOCALITY?}

1. Theory

a. A simplified quark model for non-locality.

Some non-locality of the NN interaction at short distances is expected on account of the quark structure of nucleons, that is to say, due to the nucleon compositeness. To illustrate this point, consider a very crude model, in which each nucleon is replaced by a cluster consisting of two (not three) particles. The two particles have masses $M$ and $m$, re-pectively. If there is a harmonic interaction between them, $V=\frac{2}{2} \mu \omega^{2} r^{2}$, where $\mu$ is the reduced mass $=M m /(M+m)$ and $r$ is the spacing between the particles, then the wavefunction of relative motion is a Gaussian proportional to $e^{-\mu \omega r^{2} / 2 h}=e^{-r^{2} / 2 b^{2}}$. Now consider the interaction between two clusters whose centers of mass are separated by a distance $R$. We suppose that the interaction between the particles has a range very long compared to the cluster size b, and that it involves exchanging the light particles on the two clusters. Using standard cluster model methods, it can then be shown that the interaction between the clusters is just of the non-local form discussed above:

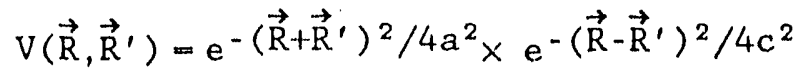

with

$$
a^{2}=2 b^{2} /\left[1+\frac{m}{M}\right]^{2}, c^{2}=2 b^{2} /\left[1+\frac{M}{m}\right]^{2}
$$

Two particular 1 imits are of interest.

i. For $m \ll M$, we obtain $a^{2}=2 b^{2}, c=0$, i.e. the interaction is local, with a

$$
V\left(\vec{R}, \vec{R}^{\prime}\right)=\mathrm{e}^{-\vec{R}^{2} / 2 b^{2}} \times \delta\left(\vec{R}-\vec{R}^{\prime}\right)
$$

range governed by the overlap of wavefunctions between the two clusters. This case corresponds to the interaction between atoms. Since the atomic nucleus has a much larger mass than an electron, the interactions between atoms are nearly local. 
i.i. For $m=M$, which more closely corresponds to the quark model of nucleons, (except for containing 2 quarks instead of 3), we find $a^{2}=c^{2}=b^{2} / 2$. For this case, the interaction is separable.

$$
V\left(\vec{R}, \vec{R}^{\prime}\right)=e^{-\left(R^{2}+R^{\prime 2}\right) / b^{2}}
$$

b. Relativistic Model

Alternatively, some non-locality also arises in relativistic nuclear models, due to relativistic retardation effects.

2. Experimental evidence: Deuteron radius There are only a few well-known examples of non-1ocal interactions known in nature. The best evidence (to date) for nonlocality of the $\mathrm{N}-\mathrm{N}$ interaction comes from the analysis of the deuteron charge radius 1 . With traditional (basically local) interactions, it is possible to fit $N-N$ scattering phase shifts very we11, but one obtains a deuteron radius about 18 larger than the empirical value. This is illustrated in Figure 1 . The problem of the deuteron radius can be easily resolved, without destroyjng the fit to phase shifts, by making the potential nonlocal ${ }^{2}$. For example, there are simple unitary transformations on the wavefunction which change the short distance behavior, but keep intact both the orthogonality properties of non-degenerate pairs of wavefunction, and the wavefunction at large distances, which is what determines the phase shifts.

\section{NON-LOCALITY AND SHELL MODEL CALCULATIONS}

\section{Two-body interaction matrix elements}

It is well known that if we use harmonic oscillator single particle wavefunctions, then the two body matrix elements for Gaussian interactions can be calculated analytically. This also holds for a non-local Gaussian, in fact, the expressions are only slightly more involved. It is readily shown that in the short range limit; i.e., when both the range a and the non-locality range $c$ are small compared to the oscillator length $b$, then the two body matrix elements reduce to those for a skyrme interaction (without density dependence). It turns out that non-locality increases the even state matrix elements, but reduces the odd state matrix elements. If $a=c$, then only matrix elements with relative orbital angular momentum zero survive. In fact, in this case, the interaction is separable. Another case of interest occurs for a long range. Here the interaction effectively reduces to a quadrupole-quadrupole interaction. Finally, for $a c=2 b^{2}$, all two body matrix elements connecting different oscillator shells vanish identically. An interaction with these (somewhat unrealistic) values of the parameters gives rise to a mean field but no splitting between states belonging to the same irreducible representation of SU(3) but different L values. 
We discuss here the Talmi integrals for non-local Gaussian interactions. Suppose we have the following non-1ocal Gaussian interaction:

$V=-V_{0} \exp -\left(\frac{\left(r+r^{\prime}\right)^{2}}{4 a^{2}}+\frac{\left(r-r^{\prime}\right)^{2}}{4 c^{2}}\right)$

in even states, and $\eta \mathrm{V}$ in ${ }^{4} C^{2} d d$ states.

Then the integrals involving relative motion can be calculated analytically. For non-local interactions, they are only slightly more complicated that for a local interaction.

We obtain:

$I_{00}=V_{0} \lambda^{3 / 2}(1-\mu)^{3 / 2}$

$\lambda=\frac{a^{2}}{a^{2}+2 b^{2}}, \mu=\frac{c^{2}}{c^{2}+2 b^{2}}$

Here is an expression for another Talmi integral:

$I_{11} / I_{00}=\eta(\lambda-\mu)$

Note that if $\lambda=\mu$, i.e. if $a-c$, (local and non-local ranges are equal), then $\mathrm{I}_{11}=0$. Similarly, all other Talmi integrals with relative angular momentum larger than 0 vanish. This is not surprising, since this case corresponds to a separable interaction, which acts only in relative s-states. Thus, for example:

$I_{22} / I_{00}=(\lambda-\mu)^{2}$

On the other hand, the Talmi integral $I_{20}$ is finite even for a separable interaction.

$I_{20} / I_{00}=(\lambda-\mu)^{2}+\frac{3}{2}(1-\lambda-\mu)^{2}$

However, there is another interesting limit, that where $\lambda+\mu=$ 1 . This corresponds to the condition $a c-2 b^{2}$. For this case, we see that $I_{20}=I_{22}$, and, more generally, $I_{N \ell}$ is independent of $\ell$. Furthermore, all off-diagonal matrix elements connecting different oscillator shells vanish.

For example,

$\mathrm{I}_{00-20} / \mathrm{I}_{00}-\sqrt{\frac{3}{2}}(1-\lambda-\mu)$

which equals 0 in this case.

It is well known that for an infinite range interaction, i.e. $\lambda=1, \mu=0$, all off-diagonal matrix elements vanish. However, as is shown here, this result holds more generally, even for a finite range interaction, provided the range of the non-locality is chosen equal to $\mathrm{c}=2 \mathrm{~b}^{2} / \mathrm{a}$. In this limit, the interaction provides a mean field, but there is no pairing. 
Finally, it should be noted that a finite range space exchange interaction, $a=r_{0}, c=0, \eta=-1,\left(\lambda=\lambda_{0}, \mu=0\right)$ has the same Talmi integrals, and thus two-body matrix elements, as a zero range, but finite non-locality range $a=0, c=r_{0}, \eta=1, \quad(\lambda=$ $0, \mu=\lambda_{0}$ ) interaction. This indicates that, to some extent the effect of non-locality can be simulated by a local. but space exchange range interaction.

\section{Connection with Skyrme Interaction}

In this talk we will restrict our consideration to a density independent interaction. For a short range interaction, i.e. with both a and $\mathrm{c} \ll \mathrm{b}$, we obtain: for the two parameters $\lambda$ and $\mu$ :

$\lambda=\frac{a^{2}}{a^{2}+2 b^{2}} \rightarrow \frac{a^{2}}{2 b^{2}}\left(1-\frac{a^{2}}{2 b^{2}}\right)$

$\mu=\frac{c^{2}}{c^{2}+2 b^{2}} \rightarrow \frac{c^{2}}{2 b^{2}}$

The Skyrme parameters are defined as follows for a local short range interaction:

$\mathrm{t}_{0}=\int_{0} \mathrm{~V}(\mathrm{r}) \quad \mathrm{d}^{3} \mathrm{r}=\mathrm{v}_{0}\left(\pi \mathrm{a}^{2}\right)^{3 / 2}$

$t_{1}=-\frac{1}{3} \int V(r) r^{2} d^{3} r=-t_{0} a^{2} / 2$

$t_{2}=\eta_{3}^{n} V(r) r^{2} d^{3} r=\eta t_{0} a^{2} / 2$

$\eta$ is the ratio of odd state to even state interaction.

$\mathrm{I}_{00}=\mathrm{V}_{0} \lambda^{3 / 2}(1-\mu)^{3 / 2} \rightarrow \mathrm{V}_{0}\left(\frac{\pi \mathrm{a}^{2}}{2 \pi \bar{b}^{2}}\right)^{3 / 2} \times\left(1-\frac{3}{4} \frac{\mathrm{a}^{2}}{\mathrm{~b}^{2}}\right)$

$I_{n o}=\int \psi_{n}(r) V\left(r, r^{\prime}\right) \psi_{0}\left(r^{\prime}\right) d^{3} r d^{3} r^{\prime}$

For a short range but non-local interaction

we must make the following changes:

in $t_{1}$, replace $a^{2}$ by $a^{2}+c^{2}$, and,

in $t_{2}$, replace $a^{2}$ by $a^{2}-c^{2}$.

Also, $I_{00}$ is now given by:

$I_{00}=V_{0} \lambda^{3 / 2}(1-\mu)^{3 / 2} \rightarrow V_{0}\left(\frac{\pi a^{2}}{2 \pi b^{2}}\right)^{3 / 2} *\left(1-\frac{3}{4} \frac{a^{2}+c^{2}}{b^{2}}\right)$

Thus non-locality increases the apparent range of the evenstate interaction, but decreases that of the odd-state interaction. 
In the skyme approximation ${ }^{3}$, the density independent part of the interaction is written as:

$\mathrm{V}(\mathrm{p}, \mathrm{r})=\mathrm{t}_{0} \delta(\mathrm{r})+\frac{1}{2}\left[\mathrm{p}^{2} \delta(\mathrm{r})+\delta(\mathrm{r}) \mathrm{p}^{2}\right] \mathrm{t}_{1}+\mathrm{p} \cdot \delta(\mathrm{r}) \cdot \mathrm{pt} \mathrm{t}_{2}$

If $t_{2}=-t_{1}$, i.e., $\eta=1$, then

$V(p, r)=t_{0} \delta(r)+\frac{1}{2}\left[p^{2} \delta(r)-2 p \cdot \delta(r) \cdot p+\delta(r) p^{2}\right] t_{1}$

$$
=t_{0} \delta(r)-\frac{1}{2}\left[\nabla^{2} \delta(r)\right] t_{1}
$$

which is just a local interaction.

The Talmi integrals for relative angular momentum zero are given as follows:

$\mathrm{I}_{\mathrm{no}} \rightarrow \psi_{\mathrm{n} 0}^{2}(0) \mathrm{t}_{0}-\psi_{\mathrm{no}}(0) \nabla^{2} \psi_{\mathrm{n} 0}(0) \mathrm{t}_{1}$

$\nabla^{2} \psi_{\mathrm{no}}(0)=-\frac{\mathrm{n}+3 / 2}{\mathrm{~b}^{2}} \psi_{\mathrm{no}}(0)$

$I_{\text {no }}=\psi_{n o}^{2}(0)\left(t_{0}+\frac{n+3 / 2}{b^{2}} t_{1}\right)=\psi_{n 0}^{2}(0) t_{0}\left(1-\frac{n+3 / 2}{b^{2}}\left(a^{2}+c^{2}\right)\right)(24)$

Also,

$$
I_{11}=\left[\nabla \psi_{11}(0)\right]^{2} t_{2}=\frac{1}{2} \eta t_{0} \frac{a^{2}-c^{2}}{b^{2}}
$$

It is interesting to list here ratios of the Talmi integrals in the Skyrme approximation:

$I_{20} / I_{00}=(\lambda-\mu)^{2}+\frac{3}{2}(1-\lambda-\mu)^{2} \rightarrow \frac{3}{2} \times\left(1-\frac{a^{2}+c^{2}}{b^{2}}\right)$

$\mathrm{I}_{22} / \mathrm{I}_{00}=(\lambda-\mu)^{2} \rightarrow \sigma\left(\alpha^{4}\right)$

$I_{00-20} / I_{00}=\sqrt{ } \frac{3}{2}(1-\lambda-\mu) \rightarrow \sqrt{\frac{3}{2}}\left(1-\frac{1}{2} \frac{a^{2}+c^{2}}{b^{2}}\right)$

$I_{11} / I_{00} \quad \eta(\lambda-\mu) \rightarrow \frac{1}{2} \eta \frac{a^{2}-c^{2}}{b^{2}}$

Finally, we discuss two possible applications of non-local interactions in the nuclear shell model.

\section{Overlap integrals}

Consider the filling of shells in the $A=100$ region. Here we have 40 to 45 protons, filling the $\mathrm{p} 1 / 2$ and $\mathrm{g} 9 / 2$ she11s, while there are 55 to 60 neutrons which fill the $g 7 / 2$ and $d 5 / 2$ she11s. There are, of course, interactions between protons and neutrons, and there is some empirical evidence from study of $\mathrm{Zr}$ and Mo isotopes that the interaction between the proton $g 9 / 2$ and neutron $\mathrm{g} 7 / 2$ shells is especially large. 4 As the neutron $g 7 / 2$ shell fills, the proton $\mathrm{g} 9 / 2$ shell drops in energy. The apparent special overlap between these shells is larger than calculated for a local zero or finite range interaction. It requires that the interaction is short range in phase space; i.e., the combined $r$ and $p$ space. We discuss here the overlap integrals for a simpler case, but one which has the essential physics, namely particles in the oscillator $(s, d)$ shell. 
As can be seen from Table 1, for a local interaction, the overlap integrals are only slightly smaller between s and d than between two particles in the sane orbits. llowever, non-locality enhances the difference, (though not by much unless the nonlocality range is large). In the extrene case of a zero range interaction with infinite range non-locality, the overlap integral vanishes unless the two particles are in the same spatial state. It is like a zero range interaction in phase space; i.e., the combined $p$ and $r$ space.

\section{Convergence of model space expansion}

For a finite non-locality range, the two body matrix elements connecting different oscillato shells disappear faster than for a purely local interaction. This should lead to more rapid convergence of shell model energies as we increase the size of the model space. Table 2 indicates how non-locality increases the rate of convergence for the simple case of ${ }^{4} \mathrm{He}$, where, in lowest approximation, the nucleons are in the oscillator ground state. Such a faster convergence of the matrix elements can be readily understood. Intermediate states correspond to larger momenta. Now, a non-local interaction can be expressed as a momentum dependent one; i.e., it falls off faster with momentum, (not just momentum transfer, as with a local interaction) then a momentum dependent one. Since the intermediate states have larger momenta, the matrix elements involving such states will be reduced.

\section{SUMMARY}

We have discussed the evidence for non-locality in the NN interaction, and the effect of a simple form of non-locality with Gaussians on nuclear shel1 model calculations. It remains to apply these ideas quantitatively to realistic NN interactions which fit scattering data, as well as the deuteron radius. of course, this requires consideration of the density dependence in the NN interaction ${ }^{5}$.

\section{REFERENCES}

1. S.Klarsfeld, J. Martore11, J.A. Oteo, M. Nishimura, and D.W. Sprung, Nucl. Phys. A456, 373 (1986)

2. M.W. Kermode, W. van Di.jk, D.W.L. Sprung, M.M. Mustafa, and S.A. Moszkowski., J. Phys. G. in press (1990)

3. D. Vautherin, and D.M. Brink, Phys. Rev. C5, 626 (1972)

4. R. Meyer, "She11. Model and Nuclear Structure", pg 227 - 250 , edited by A. Covello, World Scientific, Singapore, 1989

5. S.D. Bloom, D.A. Resler, and S.A. Moszkowski, "Contibuted Papers to the Symposium in Honor of Akito Arima: Nuclear Physics in the 1990's", Santa Fe, NM, May 1-5, 1990, p.5; also LLNL preprint, UCRL-102825, February 1990.

* Work performed under the auspices of the U.S. Department of Energy by the Lawrence Itvermore National. Laboratory under Contract W-7405-Eng-48. 
TABLE 1 .

Illustrating that non-locality leads to larger: overlap between particles in the same orbit.

\begin{tabular}{|c|c|c|c|c|c|c|}
\hline & $\gamma$ & $=\frac{a}{a^{2}+}$ & $\tilde{2} ; \mu=$ & $\frac{c^{2}}{c^{2}+2 b}$ & & \\
\hline & $\mathrm{dd}$ & a 0 & $\sqrt{\frac{2}{3} \mathrm{~b}}$ & $\sqrt{ } 2 \mathrm{~b}$ & $\sqrt{6 b}$ & $\infty$ \\
\hline c & $\mu$ & $\lambda 0$ & 0.25 & 0.5 & 0.75 & 1 \\
\hline 0 & 0 & 0.595 & 0.891 & 1.022 & 1.018 & 1.000 \\
\hline$\sqrt{\frac{2}{3} \mathrm{~b}}$ & 0.25 & 0.561 & 0.816 & 0.971 & $0.9: 8$ & 1.018 \\
\hline$\sqrt{ } 2 \mathrm{~b}$ & 0.5 & 0.496 & 0.728 & 0.921 & 0.971 & 1.022 \\
\hline$\sqrt{6 b}$ & 0.75 & 0.192 & 0.445 & 0.728 & 0.816 & 0.891 \\
\hline$\infty$ & 1.00 & 0.000 & 0.192 & 0.496 & 0.561 & 0.595 \\
\hline & ${ }^{\mathrm{ddd}}$ & a 0 & $\sqrt{\frac{2}{3} \mathrm{~b}}$ & $\sqrt{ } 2 b$ & $\sqrt{6 b}$ & $\infty$ \\
\hline c & $\mu$ & $\lambda 0$ & 0.25 & 0.5 & 0.75 & 1. \\
\hline 0 & 0 & 2.440 & 1.386 & 1.133 & 1.040 & 1.000 \\
\hline$\sqrt{\frac{2}{3} b}$ & 0.25 & 2.663 & 1.336 & 1.065 & 1.009 & 1.040 \\
\hline$\sqrt{ } 2 b$ & 0.5 & 3.745 & 1.993 & 1.316 & 1.065 & 1.133 \\
\hline$\sqrt{6 b}$ & 0.75 & 4.750 & 3.219 & 1.993 & 1.336 & 1.386 \\
\hline$\infty$ & 1.00 & 5.000 & 4.750 & 3.745 & 2.663 & 2.440 \\
\hline
\end{tabular}

\section{TABLE 2}

Illustrating that non-locality speeds up the convergence of: the model space expansion.
$\mathrm{a}=\sqrt{\frac{2}{3}} \mathrm{~b} ; \quad \lambda=\frac{\mathrm{a}^{2}}{\mathrm{a}^{2}+2 \mathrm{~b}^{2}}=\frac{1}{4} ; \quad \mu=\frac{\mathrm{c}^{2}}{\mathrm{c}^{2}+2 \mathrm{~b}^{2}} \quad 0$
$\frac{1}{4}$
$\frac{1}{2}$
$\frac{3}{4}$
$\mathrm{I}_{20} / \mathrm{I}_{O C}=\frac{3}{2}(1-\lambda-\mu)^{2}+(\lambda-\mu)^{2}$
0.91
0.38
0.16
0.25
$I_{00-20} / I_{00}=\sqrt{\frac{3}{2}}(1-\lambda-\mu)$
0.92
0.61
0.31
0.00
$I_{00-40 / I_{00}}=\sqrt{ } \frac{15}{8}(1-\lambda-\mu)^{2}$
0.77
0.34
0.08
0.00
$\mathrm{I}_{00-60 / \mathrm{I}_{00}}=\sqrt{\frac{35}{16}}(1-\lambda-\mu)^{3}$
0.62
0.18
0.02
0.00 
FIGURE 1

Calculated triplet scattering length a and deuteron radius ro for realistic NN potentials, and comparison with empirical value. From Ref. 1.

Calculated a and ${ }{ }_{D}$ for realistic potentials (Klarsfeld et al.)

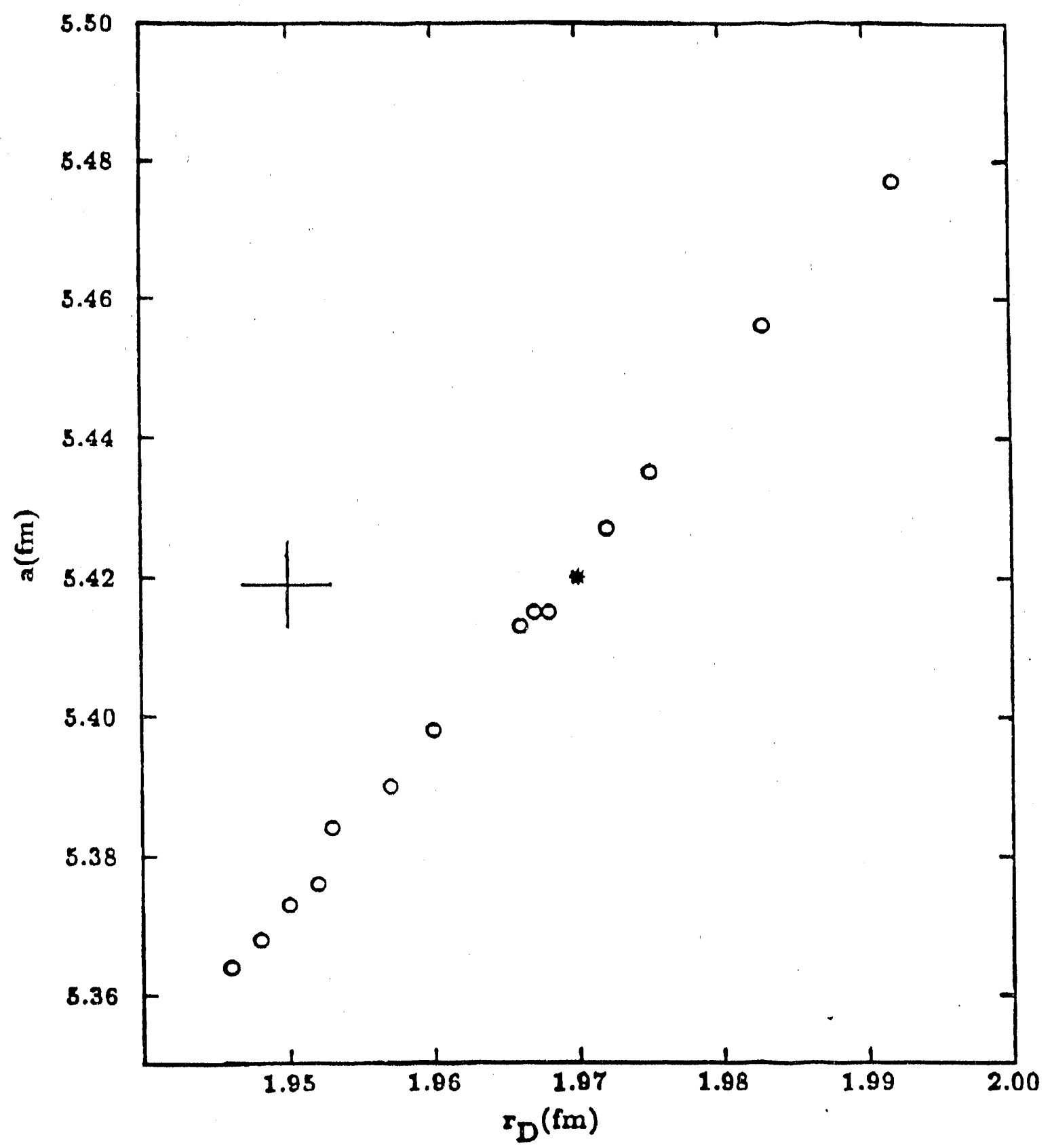



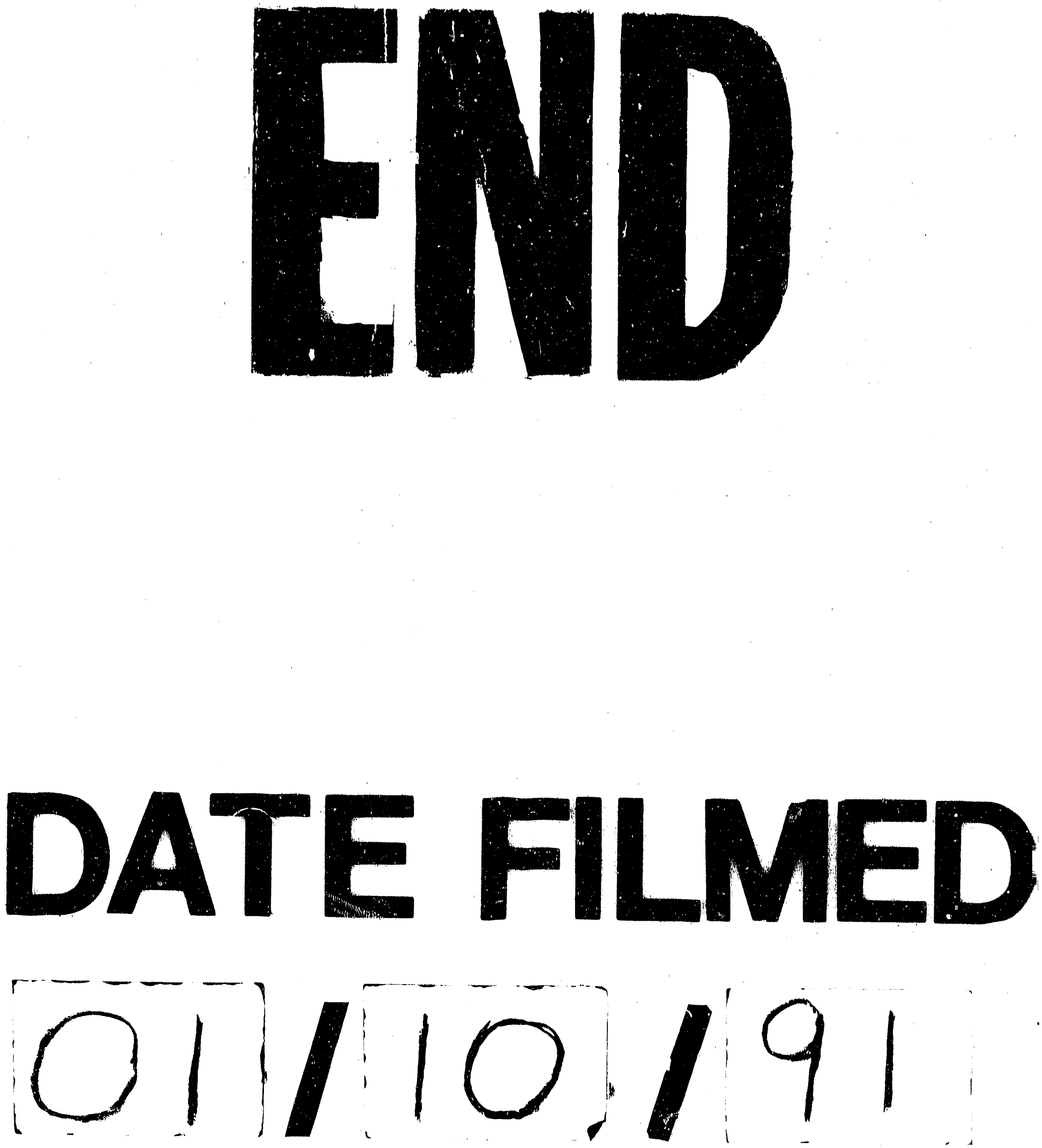